\title{
REGULAR SYNTHESES AND SOLUTIONS TO DISCONTINUOUS ODES
}

\author{
Alessia Marigo $^{1}$ And Benedetto Piccoli ${ }^{1}$
}

\begin{abstract}
In this paper we analyze several concepts of solution to discontinuous ODEs in relation to feedbacks generated by optimal syntheses. Optimal trajectories are called Stratified Solutions in case of regular synthesis in the sense of Boltyanskii-Brunovsky. We introduce a concept of solution called Krasowskii Cone Robust that characterizes optimal trajectories for minimum time on the plane and for general problems under suitable assumptions.
\end{abstract}

Mathematics Subject Classification. 49K15, 34A36, 93C15.

Received July 23, 2001. Revised February 12, 2002.

\section{INTRODUCTION}

Given an optimal control problem $\mathrm{Pb}(x)$ that depends on some free initial point $x$, one can provide a solution by means of an optimal synthesis. Roughly speaking, an optimal synthesis is a way of associating to every initial point $x$ an optimal trajectory $\gamma_{x}$. Another way of describing the solution is to define a feedback control $u(x)$ such that the corresponding trajectories are optimal. However, in most cases one has to admit discontinuous feedbacks so facing the problem of defining solutions to discontinuous ODEs. In [17] it is shown that many concepts of solution lead to not optimal trajectories or do not include all optimal trajectories. Therefore, in general, a feedback does not describe properly the solution to the family of problems $\operatorname{Pb}(x)$. However, for practical applications, the use of feedback controls is the most appropriate tool for solving many problems of different nature such as optimal stabilization [12,14], trajectory tracking [2] and motion planning [15]. Hence, it is of fundamental importance the question of providing a suitable definition of solution, to discontinuous ODEs, that determines exactly the set of optimal trajectories from the discontinuous feedback.

The difficulty of the problem is illustrated by the Example 5.3 in [17] exibiting an optimal synthesis whose corresponding feedback admits classical solutions (i.e. smooth solutions satisfying the equation at each time) that are not optimal, even worse, some of them do not reach the target. Hence any generalized concept of solution, for which classical solutions are admissible, is not proper.

We consider many concepts of solution to discontinuous ODEs, some already introduced in the literature and some new, and also analyze the concept of regular synthesis in the sense of Boltyanskii-Brunovsky (see $[3,6]$ ). The latter is based on the concept of stratification and is the more appropriate definition of synthesis to discuss the properties of the set of solutions to the corresponding discontinuous feedback. For reference about the construction of stratifications see [9] and [10]. In [17] a more general definition of synthesis is introduced, which is suitable for a general sufficiency theorem, but such syntheses are not necessarily generated by a feedback.

None of the introduced concepts of solution works properly for every optimal control problem. This is not too surprising since, even the description of optimal synthesis for smooth systems, is usually given only under generic assumptions, see $[5,11,16]$. If no generic assumption is taken, then any kind of singularity may occur

Keywords and phrases: Optimal control, regular synthesis, discontinuous ODEs.

1 IAC-CNR, Viale Policlinico 137, 00161 Roma, Italy; e-mail: marigo,piccoli@iac.rm.cnr.it 
in the optimal synthesis with no hope of controlling the behavior of generalized solutions to the corresponding discontinuous feedback. Therefore, we take the point of view of studying systems in generic position.

The first results regard minimum time for single input systems on the plane. The syntheses with point target were classified in [5,18]. Moreover, the set of extremals was studied in details in [4]. Under generic assumptions, only 5 types of singular curves and 17 types of singular points can appear, see [16]. Thus we can analyze the generic case thanks to this finite list of possible singularities. The results are summurized in Theorem 2, where we show that the concept of Krasowskii Cone Robust Solution, introduced in Section 3, individuates optimal trajectories. A Krasowskii solution is cone robust if it is robust with respect to perturbations of the initial point along some cone with nonempty interior. Notice that, the importance of Krasowskii Cone Robust Solutions is not bounded to the property of describing optimal trajectories, but shows up also in numerical implementation issues. Indeed, numerical schemes, introducing rounding errors, are able to generate only "observable" trajectories, that is trajectories robust with respect to perturbations of initial data.

We also describe a new definition of regular synthesis, already introduced in [13], that is modeled on Boltyanskii-Brunovsky definition, and conditions such that Krasowskii Cone Robust solutions coincide with solutions of the optimal synthesis. These conditions are again in the spirit of generic assumptions. Moreover, each assumption is discussed with examples that illustrate the effects on the shape of the optimal synthesis.

Section 2 is dedicated to basic definitions and to the definition of regular synthesis. In Section 3 we review some concepts of solutions to discontinuous ODEs and introduce new ones. Section 4 is dedicated to minimum time on the plane: we prove that Krasowskii Cone Robust Solutions coincide with optimal ones. Finally, in Section 5, we give sufficient conditions on a regular synthesis for a general system to have the same conclusion. Moreover we show the properties of Krasowskii extremal solutions.

\section{BASIC DEFINITIONS}

We indicate by $B_{n}$ the unit ball in $\mathbb{R}^{n}$. Given a set $A \subset \mathbb{R}^{n}$ we denote by $\operatorname{cl}(A)$ the closure of $A$, by $\operatorname{Int}(A)$ its interior and by $\overline{c o}(A)$ the closed convex hull that is

$$
\overline{\mathrm{co}}(A) \stackrel{\text { def }}{=} \operatorname{cl}\left\{\sum \lambda_{i} x_{i} ; x_{i} \in A, \lambda_{i} \in \mathbb{R}, \lambda_{i} \geq 0, \sum \lambda_{i}=1\right\} .
$$

A cone $\mathcal{K} \subset \mathbb{R}^{n}$ is a set such that for every $\lambda>0$ we have $\lambda \mathcal{K} \subset \mathcal{K}$. A set $A \subset \mathbb{R}^{n}$ is convex if $\lambda x+(1-\lambda) y \in A$ for every $x, y \in A$ and $0 \leq \lambda \leq 1$. A point $x$ of a convex set $A$ is said extremal if whenever $x=\lambda y_{1}+(1-\lambda) y_{2}$, with $y_{1}, y_{2} \in A$ and $0<\lambda<1$, it follows $x=y_{1}=y_{2}$.

A multifunction $V$ is a map from a set $A$ into the set of subsets of another set $A^{\prime}$, thus $V(x) \subset A^{\prime}$ for every $x \in A$.

On the set of subsets of $\mathbb{R}^{n}$ we consider the Hausdorff distance $d\left(A, A^{\prime}\right)=\sup \left\{d\left(x, A^{\prime}\right), d\left(A, x^{\prime}\right): x \in A, x^{\prime} \in\right.$ $\left.A^{\prime}\right\}$, where $d\left(x, A^{\prime}\right)=\inf \left\{d\left(x, x^{\prime}\right): x^{\prime} \in A^{\prime}\right\}$ and similarly for $d\left(A, x^{\prime}\right)$. Assume $V$ is a multifunction from $\mathbb{R}^{n}$ to $\mathbb{R}^{n^{\prime}}$. Then $V$ is continuous if it is continuous for the Hausdorff distance. We say that the multifunction $V$ is upper semicontinuous at $x$ if for every $\varepsilon>0$ there exists $\delta>0$ such that $V(y) \subset V(x)+\varepsilon B_{n^{\prime}}$ for every $|y-x|<\delta$.

Given a multifunction $V$ from $\mathbb{R}^{n}$ to $\mathbb{R}^{n}$ we consider the corresponding differential inclusion that is

$$
\dot{x} \in V(x) .
$$

A solution (in Caratheodory sense) to (1) is an absolutely continuos function $x$ such that $\dot{x}(t) \in V(x(t))$ for almost every $t$ in the domain of $x$. If $V$ has convex values then we also consider the differential inclusion

$$
\dot{x} \in \operatorname{ext} V(x)
$$

where ext $V(x)$ is the set of extremal points of $V(x)$. 
We consider a minimization problem of the type:

$$
\operatorname{Pb}(\bar{x})=\left\{\begin{array}{l}
\dot{x}=f(x, u) \\
x(0)=\bar{x}, \quad x(T) \in \mathcal{T} \\
\left.\min \int_{0}^{T} L(x(t), u(t))\right) \mathrm{d} t+\Phi(x(T))
\end{array}\right.
$$

with $x \in \mathbb{R}^{n}, u \in U$ where $U \subset \mathbb{R}^{m}$, compact, is the control set, $f: \mathbb{R}^{n} \times U \rightarrow \mathbb{R}^{n}$ is continuous and continuously differentiable w.r.t. $x, \bar{x} \in \mathbb{R}^{n}$ is the initial condition, $\mathcal{T} \subset \mathbb{R}^{n}$, smooth manifold, is the target, $L: \mathbb{R}^{n} \times U \rightarrow \mathbb{R}^{n}$, the Lagrangian, is continuous and continuously differentiable w.r.t. $x$ and the final cost $\Phi: \mathbb{R}^{n} \rightarrow \mathbb{R}^{n}$ is differentiable. An admissible control is a measurable map $u:[0, T] \rightarrow U$ such that $(t, x) \mapsto(f(x, u(t)), L(x, u(t)))$ has Jacobian w.r.t. $x$ uniformly bounded on compact sets. The minimization is taken over all trajectories, corresponding to admissible controls, satisfying the boundary conditions $x(0)=\bar{x}$ and $x(T) \in \mathcal{T}$.

A way of describing a solution to the collection of problems $\{\operatorname{Pb}(\bar{x}): \bar{x} \in \Omega\}$, where $\Omega$ is a subset of $\mathbb{R}^{n}$, is to provide an optimal synthesis on $\Omega$.

Definition 1. An optimal synthesis on $\Omega$ is a collection of trajectories $\left\{\gamma_{x}: x \in \Omega\right\}$ such that $\gamma_{x}$ is optimal, i.e. solves the problem $\mathrm{Pb}(x)$.

\subsection{Regular synthesis}

To define a regular synthesis we first need to recall the definition of Withney stratified set.

Definition 2. Let $M$ be a subset of $\mathbb{R}^{n}$ and assume $M=\cup_{j \in J} M_{j}$, where $J \subset \mathbb{N}$ and $M_{j}$ are disjoint nonemtpy connected embedded $\mathcal{C}^{1}$ submanifolds of $\mathbb{R}^{n}$. Then $M$ is a Withney stratified set if the collection $\mathcal{P}:=\left\{M_{j}\right\}_{j \in J}$, called the stratification of $M$, is locally finite and the following holds.

- If $M_{k} \cap \operatorname{cl}\left(M_{j}\right) \neq \emptyset(j \neq k)$ then $M_{k} \subset \partial M_{j}$ and $\operatorname{dim}\left(M_{k}\right)<\operatorname{dim}\left(M_{j}\right)$.

- Let $x_{n}, y_{n} \in M_{j}, n \in \mathbb{N}, x_{n}, y_{n} \rightarrow \bar{x} \in M_{k} \subset \operatorname{cl}\left(M_{j}\right)$ and denote by $\ell_{n}$ the direction in $\mathbb{R}^{n}$ containing the segment joining $x_{n}$ with $y_{n}$. If $T_{x_{n}} M_{j} \rightarrow T$ (in the Grassmannian) and $\ell_{n} \rightarrow \ell$, then $\ell \subset T$ and $T_{\bar{x}} M_{k} \subset T$.

We define the dimension of $M$ by $\operatorname{dim}(M)=\max _{j} \operatorname{dim}\left(M_{j}\right)$.

We introduce the concept of regular synthesis that is modeled on the definitions given by Boltyanskii and Brunovsky, see $[3,6]$. For simplicity, we consider the case of optimal synthesis with target a point that we may assume to be the origin.

Definition 3. Let $\Omega$ be an open set containing the origin. A regular synthesis on $\Omega$ is a 6 -tuple $\Xi=$ $\left(\mathcal{P}, \mathcal{P}_{1}, \mathcal{P}_{2}, \Pi, \Sigma, u\right)$ such that

(RS.1) $\Omega$ is a Withney stratified set with stratification $\mathcal{P} .\{0\} \in \mathcal{P}$. The elements of $\mathcal{P}$ are called "cells";

(RS.2) $\mathcal{P} \backslash\{\{0\}\}$ is the disjoint union of $\mathcal{P}_{1}$ (the set of "type I cells") and $\mathcal{P}_{2}$ (the set of "type II cells");

(RS.3) the feedback $u:\left\{x: x \in P_{1} \in \mathcal{P}_{1}\right\} \rightarrow U$ and $\Pi: \mathcal{P}_{1} \rightarrow \mathcal{P}$ are maps, $\Sigma: \mathcal{P}_{2} \rightarrow \mathcal{P}_{1}$ is a multifunction, with non empty values, such that the following properties are satisfied:

(RS.A) the function $u$ is of class $\mathcal{C}^{1}$ on each cell;

(RS.B) if $P_{1} \in \mathcal{P}_{1}$ then $f(x, u(x)) \in T_{x} P_{1}$ (the tangent space to $P_{1}$ at $x$ ) for every $x \in P_{1}$. In addition, for each $x \in P_{1}$, if we let $\xi_{x}$ be the maximally defined solution to the initial value problem

$$
\dot{\xi}=f(\xi, u(\xi)), \quad \xi(0)=x, \xi \in P_{1}
$$

and define $t_{x}=\sup \operatorname{Dom}\left(\xi_{x}\right)$, then the limit $\xi_{x}\left(t_{x}-\right) \stackrel{\text { def }}{=} \lim _{t \uparrow t_{x}} \xi_{x}(t)$ exists and belongs to $\Pi\left(P_{1}\right)$; 
(RS.C) if $P_{2} \in \mathcal{P}_{2}$, then for each $x \in P_{2}$ and $P \in \Sigma\left(P_{2}\right)$ there exists a unique curve $\xi_{x}^{P}:\left[0, t_{x}^{P}[\rightarrow \Omega\right.$ such that the restriction of $\xi_{x}^{P}$ to $] 0, t_{x}^{P}[$ is a maximally defined integral curve of the vector field $f(\cdot, u(\cdot))$ on $P$, and $\xi_{x}^{P}(0)=x$;

(RS.D) on every cell $P_{1} \in \mathcal{P}_{1}, x \rightarrow t_{x}$ is a continuously differentiable function, and $(t, x) \rightarrow \xi_{x}(t),(t, x) \rightarrow$ $u_{x}(t) \stackrel{\text { def }}{=} u\left(\xi_{x}(t)\right)$ are continuously differentiable maps on the set

$$
E(P) \stackrel{\text { def }}{=}\left\{(t, x): x \in P_{1}, t \in\left[0, t_{x}\right]\right\}
$$

in the sense that they can be prolonged to maps of class $\mathcal{C}^{1}$ on some open subset of $\mathbb{R} \times P_{1}$ containing $E\left(P_{1}\right)$. If $P_{2} \in \mathcal{P}_{2}$ the same holds for every $t_{x}^{P}, \xi_{x}^{P}, u_{x}^{P}$, with $P \in \Sigma\left(P_{2}\right)$;

(RS.E) for every $x \in \Omega \backslash\{0\}$, if we let $\tilde{\xi}_{x}$ denote a curve, starting at $x$, obtained by piecing together the trajectories on every single cell, then $\tilde{\xi}_{x}$ ends at the origin in finite time.

Definition 4. The trajectories $\tilde{\xi}_{x}$ of $(R S 3 . E)$ are called Stratified Solutions of $\Xi$.

Remark. The original definitions of Boltyanskii and Brunovsky are different between them and from our definition. For a comparison of the two concepts we refer to [17]. In our definition we first ask a more restrictive assumption on the stratification of $\Omega \backslash\{0\}$, namely we ask the stratification of this set to be a Withney stratification. The definition of Brunovsky only asked for a stratification, that is only the first condition of Definition 2 is required, and the definition of Boltyanskii did not even ask for the partition $\mathcal{P}$ to be locally finite.

Another difference is that we do not ask for $\tilde{\xi}_{x}$ to have a finite number of switchings, that is to pass from one cell to another a finite number of times.

In Boltyanskii definition a set $N$ exists such that from every point of $N$ more than one optimal trajectory can start. This was done to include the case of overlaps (see Sect. 5.3). This set $N$ was not a cell of type I or II. While in Brunovsky there is not such set and the overlaps are admitted as II cells for which we must decide which cell of type I we are going to enter. We choose not to have such a set $N$ but we allow the map $\Sigma$ of (RS.3) to be multivalued (while in Boltyanskii and Brunovsky definitions it was single valued). So we have that overlaps are II cells but we allow all optimal trajectories to be included as Stratified solutions.

Hence we introduce another concept of solution:

Definition 5. If we set the multifunction $\Sigma$ of $(R S .3)$ of Definition 3 to be single valued then we call the corresponding trajectories $\tilde{\xi}_{x}$, of $(R S . E)$, Brunovsky Stratified solutions to $\Xi$.

\section{Solutions to Discontinuous ODEs}

In this section we describe various concepts of solution to discontinuous ODEs, which are used in the following sections for regular synthesis.

We consider an ODE with a discontinuous righthand side:

$$
\dot{x}=v(x), \quad x \in \mathbb{R}^{n},
$$

where $v: \mathbb{R}^{n} \rightarrow \mathbb{R}^{n}$ is a measurable bounded function. In our case $v$ denotes $f(x, u(x))$. We start recalling the definition of Caratheodory solution.

Definition 6. A Caratheodory solution to (4) is an absolutely continuous function $x:[0, T] \rightarrow \mathbb{R}^{n}$ that verifies (4) for almost every $t \in[0, T]$.

Next we use the theory of differential inclusions to introduce the concepts of Krasowskii and Filippov solution, see $[8]$. 
Definition 7. Given a measurable bounded function $v: \mathbb{R}^{n} \rightarrow \mathbb{R}^{n}$ we associate two multifunctions $V_{K}$ and $V_{F}$ in the following way:

$$
\begin{gathered}
V_{K}(x)=\bigcap_{\delta>0} \overline{\mathrm{co}} v\left(x+\delta B_{n}\right) \\
V_{F}(x)=\bigcap_{\delta>0} \bigcap_{\operatorname{meas}(N)=0} \overline{\mathrm{co}} v\left(\left(x+\delta B_{n}\right) \backslash N\right)
\end{gathered}
$$

where $N \subset \mathbb{R}^{n}$, meas is the Lebesgue measure on $\mathbb{R}^{n}$.

Notice that if $v$ is continuous at $x$ then $V_{K}(x)=V_{F}(x)=v(x)$.

Definition 8. A Krasowskii (resp. Filippov) solution to $\dot{x}=v(x)$ is a solution to the differential inclusion $\dot{x} \in V_{K}(x)\left(\operatorname{resp} \dot{x} \in V_{F}(x)\right)$.

Since $v$ is bounded, the multifunctions $V_{K}$ and $V_{F}$ are upper semicontinuous, with compact convex values. It follows (see [1]) the following:

Proposition 1. Since $v$ is bounded, for every $x \in \mathbb{R}^{n}$ and every $T>0$, the set of Krasowskii (Filippov) solutions defined on $[0, T]$ starting at $x$ is a nonempty, connected, compact subset of $\mathcal{C}\left([0, T], \mathbb{R}^{n}\right)$.

Remark. The concept of Krasowskii solution is more appropriate for regular synthesis than the one of Filippov. Indeed, if we are on a cell $P$ of type I with positive codimension then the multifunction $V_{F}$ contains only the limiting direction from neighboring cells of maximal dimension. Thus the feedback $v(x)$, for $x$ in $P$, is not in general contained in $V_{F}(x)$. On the contrary, we always have $v(x) \in V_{K}(x)$. Hence in Section 5 we restrict our analysis to Krasowskii solutions.

For our purposes we need to select among Krasowskii solutions those that present some robustness with respect to perturbations of initial data. We thus introduce the following:

Definition 9. A Krasowskii solution $x:[0, T] \rightarrow \mathbb{R}^{n}$ is called Krasowskii Cone Robust if, for every $t \in[0, T]$, there exist $a(x(t)) \geq a>0$ and a cone $\mathcal{K}(x(t)) \subset \mathbb{R}^{n}$, called Robustness Cone, with $\operatorname{Int}(\mathcal{K}(x(t))) \neq 0$, such that the following holds. The multifunction $t \mapsto \mathcal{K}(x(t)) \cap B_{n}$ is continuous. Moreover, for every $y_{n} \rightarrow x(t)$, $y_{n}=x(t)+\epsilon_{n} w+o\left(\epsilon_{n}\right)$, with $w \in \mathcal{K}(x(t))$ and $\epsilon_{n} \rightarrow 0$, and every $x_{n}(\cdot)$, Krasowskii solutions with $x_{n}(t)=y_{n}$, we have that $x_{n}(\cdot)$ converge to $x$ uniformly on $[t, \min \{t+a(x(t)), T\}]$. If $\mathcal{K}(x(t))=\mathbb{R}^{n}$ for every $t$, then we say that $x$ is a Krasowskii Robust solution. Similar definitions are given for Filippov solutions.

Remark. Notice that we ask $\mathcal{K}$ to depend continuously on $x(t)$. This might prevent the choice of the maximal Cone of Robustness. However, this definition guarantees results as the next theorem.

We have the following:

Theorem 1. Let $x:[0, T] \rightarrow \mathbb{R}^{n}$ be a Krasowskii solution and assume that there exists a cone with non empty interior $\mathcal{K} \subset \mathbb{R}^{n}$ s.t. $v$ is smooth on $A \stackrel{\text { def }}{=}\{x(t)+w: t \in[0, T], w \in \mathcal{K}\}$ and $\dot{x}(t)=\lim _{y \rightarrow x(t), y \in A} v(y)$. Then $x(\cdot)$ is Krasowskii Cone Robust with robustness cone $\operatorname{Int}(\mathcal{K})$.

Proof. Let $y_{n}$ be a sequence converging to $x(t)$ along a direction $w \in \operatorname{Int}(\mathcal{K})$. Since $v$ is smooth on $A$, there exists a unique solution $x_{n}(\cdot)$, defined in a neighborhood of $t$, such that $x_{n}(t)=y_{n}$ and $\dot{x}_{n}(s)=v\left(x_{n}(s)\right)$, as long as such a solution remains inside the set $A$. Let $w(s)$ be the solution to the variational equation

$$
\dot{w}(s)=\nabla v(x(s)) \cdot w(s),
$$

with initial data $w(t)=w$. For $\epsilon$ sufficiently small $w(s) \in \operatorname{Int}(\mathcal{K})$ for all $s \in[t, t+\epsilon]$. The continuous dependence from initial data of solutions to the equation $\dot{y}=v(y)$ ensures that $x_{n}(s) \in x(s)+\mathcal{K}$ for $n$ sufficiently big and $s \in[t, t+\epsilon]$. Since $y_{n}$ converges to $x(t)$ and $v\left(x_{n}(s)\right)$ converges to $\dot{x}(s)$ we get the conclusion. 
We will also consider solutions taking extremal velocities.

Definition 10. A Krasowskii Extremal solution is a solution to the differential inclusion $\dot{x} \in$ ext $V_{K}(x)$. Similarly for Filippov solutions.

Finally we recall the definition of "sample and hold" solutions introduced in [7].

Definition 11. Given $[0, T]$ and a partition $0=t_{0}<t_{1}<\cdots<t_{m}=T$ an Euler solution is a solution for $\dot{x}(t)=v\left(x\left(t_{i}\right)\right)$ for every $t \in\left[t_{i}, t_{i+1}[, i=0, \ldots, N-1\right.$. A CLSS solution $x$ is a uniform limit of Euler solutions corresponding to partitions such that $\sup \left\{\left|t_{i+1}-t_{i}\right|: i=1, \ldots, N-1\right\}$ tends to zero.

In next section we use the following symbols for solutions to discontinuous ODEs:

- $S t$ stands for Stratified solutions and $B-S t$ stands for Brunovsky Stratified solutions;

- $C a$ stands for Caratheodory solutions;

- $K r(F i)$ stands for Krasowskii (Filippov) solutions;

- $\operatorname{KrCR}(\mathrm{FiCR})$ stands for Krasowskii (Filippov) Cone Robust solutions;

- $K r R(F i R)$ stands for Krasowskii (Filippov) Robust solutions;

- $K r E$ (FiE) stands for Krasowskii (Filippov) Extremal solutions.

\section{Planar Synthesis}

In this section we consider the problem of minimum time stabilization to the origin for the control system:

$$
\dot{x}=F(x)+u G(x)
$$

where $x \in \mathbb{R}^{2},|u| \leq 1$ and $F, G$ are smooth vector fields on $\mathbb{R}^{2}$ with bounded derivatives and we assume $F(0)=0$. Thus, for every $x \in \mathbb{R}^{2}$, we have the problem $\mathrm{Pb}(x)$ of steering $x$ to the origin in minimum time. Given $\tau>0$, the controllable set $C(\tau)$ in time $\tau$ is the set of all points that can be steered to the origin by a trajectory of (5) in time less than or equal to $\tau$. An optimal synthesis exists on $C(\tau)$ for generic smooth planar systems, see $[5,16]$ (for analytic systems see [18]). Moreover, the optimal synthesis is a regular synthesis and the generic singularities of these syntheses were classified in [16]. The classification is topological and two points are equivalent if the synthesis, near these points, has the same shape.

The generic singular curves are of five types: $Y 1, Y 2$ (or equivalently $X 1, X 2$ ), $C, S$ and $K$. Each singular point arises from the intersection of two singular curves. We use the notation of [16] so a point is said type of $\left(F_{1}, F_{2}\right)$ if it is intersection of two singular curves of type $F_{1}$ and $F_{2}$. There are 17 generic equivalence classes of singular points: $(X, Y),(Y, C)_{1,2,3},(Y, S),(Y, K)_{1,2,3},(C, C)_{1,2},(C, S)_{1,2},(C, K)_{1,2},(S, K)_{1,2}$ and $(K, K)$. We represent in Figures 1 and 2 the optimal synthesis near each singularity.

Each open region is covered by bang-bang trajectories that correspond either to constant control $u \equiv 1$ or to constant control $u \equiv-1$. The singular curves $Y 1$ and $Y 2$ are trajectories, hence cells of type I, corresponding to constant control +1 and are equivalent to singular curves $X 1$ and $X 2$, respectively, that are trajectories corresponding to constant control $-1 . S$ is also a trajectory but corresponds to a control $u \notin\{-1,+1\}$ (generically for almost all times). The curves $C$ and $K$ are not trajectories and are cells of type II.

In the rest of the section we analyze the properties of solutions to the corresponding discontinuous optimal feedback, according to the definitions of solutions introduced in the previous section. CLSS solutions are discussed in Section 4.3, while Extremal solutions are examined in some remarks.

For each singularity we consider all solutions passing through it, not only those starting from a point of the singularity. Thus robustness is considered for all these trajectories.

\subsection{Singular curves}

\section{Curves $X 1, X 2$ and $C$}

In these cases all solutions coincide. 


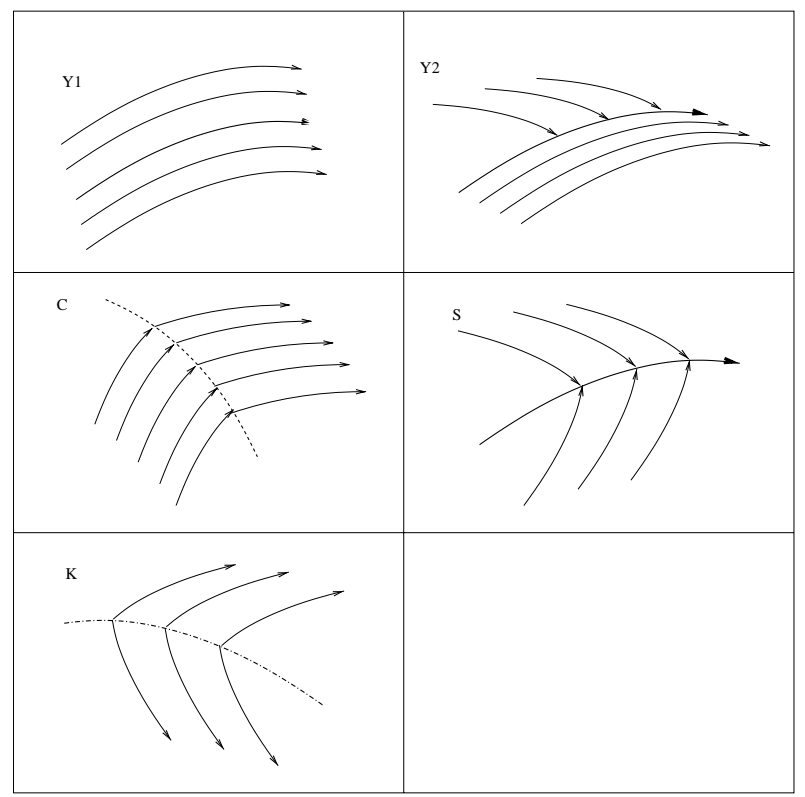

Figure 1. Singular curves.

\section{Curve $S$}

In this case we have that $B-S t=S t=C a$. Since the system is single input we also have that $V_{K}=V_{F}$ and $F i=K r=C a$ and all solutions coincide.

Remark. In this case the set of Extremal solutions is empty. If otherwise the system is not single input then it can happen that $V_{K} \supset V_{F}$ with strict inclusion. More precisely, given $x \in S$, if $v_{1}(x)$ and $v_{2}(x)$ are the limiting direction of $F+u G$ from the left and the right of $S$, we may have that $F(x)+u(x) G(x)$ is not in the segment with end points $v_{1}(x), v_{2}(x)$. Therefore $K r=F i \cup C a$, i.e. while the set of Filippov solutions does not contain the Stratified ones, the set of Krasowskii solutions is comprised also of those which are not stratified. In this case the Stratified solutions $S t$ coincide exactly with $K r E$ solutions.

\section{Curve $K$}

Here $V_{K}=V_{F}$ hence $K r=F i \supset C a$. Indeed there are Krasowskii (Filippov) solutions that run on the curve $K$, while Caratheodory solutions exit the curve $K$. Moreover $C a=S t \supset B-S t$. The last relation comes from the fact that $K$ is a II cell and $B-S t$ solutions exit only towards one of the two sides of $K$. The set of $\operatorname{KrCR}(\mathrm{FiCR})$ solutions coincide with $S t$ solutions where for each curve the corresponding cone of robustness is contained on the side (w.r.t. $K$ ) where the curve points into. No stratified solution is robust, that is the Robustness Cone can not be taken to be the whole space.

Remark. Observe that solutions corresponding to the extremal points of $V_{K}\left(V_{F}\right)$ coincide with $S t$.

\subsection{Singular points}

We analyze the case $(C, K)_{1}$ in detail, because at this singular point there is a non trivial behavior of solutions. The other points are analyzed more quickly since many arguments can be repeated in almost the same manner.

Point $(C, K)_{1}$

Let $x$ be the $(C, K)_{1}$ point. There are two Stratified solutions through $x$. Both are bang-bang, i.e. corresponding to control \pm 1 : one corresponds to constant control near $x$, the other switches from control +1 to 


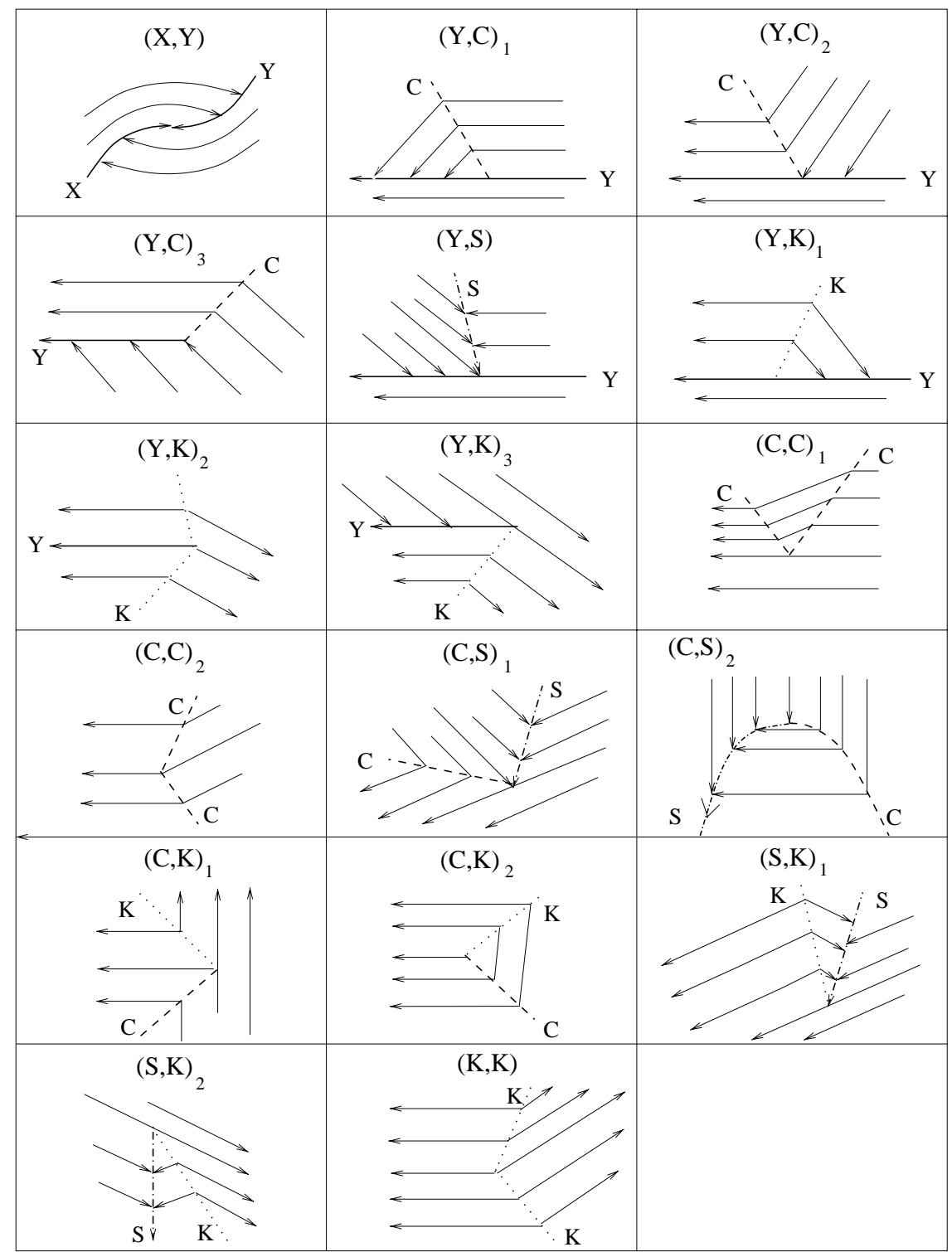

FiguRE 2. Singular points.

control -1 (or vice versa) at $x$. The set of Krasowskii (Filippov) Solutions includes trajectories that reach $x$ and then run on the curve $K$. Thus $K r=F i \supset C a=S t \supset B-S t$ (see also the discussion of $K$ curves).

The two stratified solutions are also Krasowskii Cone Robust Solutions indeed, referring to Figure 2 , let $\Omega_{1}$ $\left(\Omega_{2}\right)$ be the bigger (smaller) region enclosed by $C$ and $K, \gamma_{1}\left(\gamma_{2}\right)$ the Stratified solutions that, after $x$, run on $\Omega_{1}\left(\Omega_{2}\right)$. Then $\gamma_{1}$ is stable for any perturbation to its right, while $\gamma_{2}$ is stable at $x$ for any perturbation in $\Omega_{2}$. However neither $\gamma_{1}$ nor $\gamma_{2}$ is Krasowskii Robust. Indeed perturbation of $\gamma_{1}$ to its left do not converge to $\gamma_{1}$ and the same for $\gamma_{2}$ and perturbations to its right.

Finally $K r C R=F i C R=S t \supset K r R=F i R=\emptyset$. 
Point $(X, Y)$

We have $V_{K}=V_{F}= \pm G$. It is easy to check that all solutions coincide.

Remark. The set of Extremal solutions is empty.

Points $(Y, C)_{1},(Y, C)_{2}$ and $(Y, C)_{3}$

Here $V_{K}=V_{F}$ and $K r=F i=C a=S t$. Moreover every Stratified solution is also robust, i.e. St $=K r R$ $(S t=F i R)$.

Point $(Y, S)$

Since the system is single input, $V_{K}=V_{F}$ and $K r=F i=C a=S t$. Stratified solutions are also robust and all solutions coincide.

Point $(Y, K)_{1}$

Here we refer to the discussion we have done for the case of the singular curve $K$. Also in this case we have that $V_{K}=V_{F}$ and $K r=F i \supset C a$. Moreover $C a=S t \supset B-S t$. The set of $K r R(F i R)$ solutions coincide with $S t$ solutions.

Points $(Y, K)_{2}$ and $(Y, K)_{3}$

These two cases differ from the case $(Y, K)_{1}$ in the fact that robustness of Stratified solutions is only with respect to a cone and not with respect to the whole space.

Points $(C, C)_{1}$ and $(C, C)_{2}$

In these two cases all solutions coincide.

Points $(C, S)_{1}$ and $(C, S)_{2}$

Here we refer to the discussion we have done for the case of the singular curve $S$. Since the system is single input all solutions coincide.

Point $(C, K)_{2}$

This case differs from the case $(C, K)_{1}$ because the trajectory passing through the singular point is robust. Hence $K r=F i \supset C a=S t, S t \supset B-S t$ and $K r R=F i R=S t$.

Point $(S, K)_{1}$

On the curve $K$, we have that $K r=F i \supset C a=S t$ and $S t \supset B-S t$ and, on curve $S$, we have that $K r=F i=S t$. Moreover the Stratified solutions are Krasowskii Cone Robust and some are also Krasowskii Robust. Hence $\operatorname{KrCR}=\mathrm{FiCR}=$ St.

Point $(S, K)_{2}$

This case differs from the previous one because there is no Krasowskii Robust solution passing through the singular point. But also in this case $K r C R=F i C R=S t$.

Point $(K, K)$

The analysis of this case is consequence of the properties of the curve $K$ only. Krasowskii and Filippov solutions coincide and strictly contain Caratheodory and Stratified solutions, i.e. Fi $=K r \supset C a=S t \supset B-S t$. Moreover Stratified solutions are only Cone Robust, i.e. $S t=K r C R=F i C R$. 


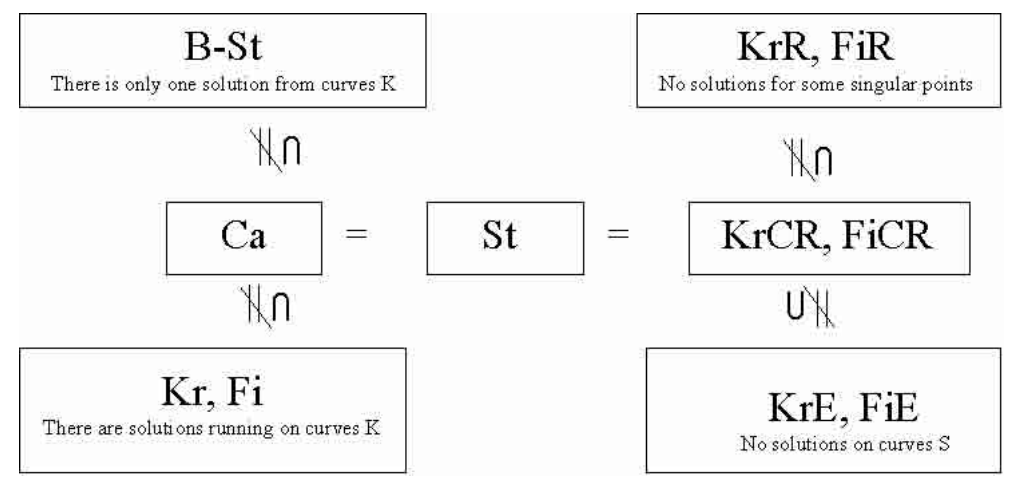

FigURE 3. Solutions to planar synthesis.

\subsection{Planar stratified solutions}

From the above analysis on generic singularities we obtain the following

Theorem 2. Consider the minimum time problems $\mathrm{Pb}(x), x \in \mathbb{R}^{2}$, with initial point $x$ and final point the origin for the control system (5). Then, given $\tau>0$, under generic conditions there exists an optimal synthesis on $C(\tau)$ such that the set of Stratified solutions coincide with the set of Krasowskii Cone Robust solutions. More precisely we have the situation of Figure 3.

Remark. Notice that many concepts of solutions introduced in the previous section do not work to isolate Stratified solutions. The situation of CLSS solutions is quite special. Consider a singular curve of type $K$. Then there is no feedback defined on it. This is not a problem to define solutions in Krasowkii, Filippov or Caratheodory sense. However for CLSS we need the control at initial point to be specified. Thus we have to define a control on the singular curve. Whatever control we define on the singular curve, the corresponding set of CLSS solutions can not encompass both Stratified solutions starting from a point $x$ of $K$, unless the following occurs. Let $\mathcal{O}$ be a neighborhood of $x$ such that $\left(\mathbb{R}^{2} \backslash K\right) \cap \mathcal{O}$ has two connected components $\Omega_{1}, \Omega_{2}$. There exists $w \in \mathbb{R}^{2}$ and a control $u \in U$ such that $w=F(x)+u G(x)$ and for every $\delta>0$ there exist $\lambda_{1}, \lambda_{2}$, with $\left|\lambda_{i}\right|<\delta$ and $x+\lambda_{i} w \in \Omega_{i}, i=1,2$. However, the latter condition is not generic.

\section{General CASe}

We introduce in Section 5.1 the definition of Krasowskii Admissible Regular Synthesis and discuss it. Then in Section 5.2 we give our main result ensuring that, for Krasowskii Admissible Regular Synthesis, the set of Stratified solutions coincide with the set of Krasowskii Cone Robust solutions. Moreover we illustrate the role of Caratheodory solutions. Section 5.3 is dedicated to special II cells called overlaps. In Section 5.4 the assumptions of Theorem 3 are analysed and some properties of Stratified solutions are given. Finally, Section 5.5 is dedicated to Krasowskii Extremal solutions.

\subsection{Krasowskii admissible regular synthesis}

We introduce the notion of Krasowskii admissible regular synthesis. This definition contains some assumptions additional to those of regular synthesis, in order to guarantee that Krasowskii Cone Robust solutions coincide with Stratified ones. We first need some more notation.

Definition 12. Consider a regular synthesis $\Xi=\left(\mathcal{P}, \mathcal{P}_{1}, \mathcal{P}_{2}, \Pi, \Sigma, u\right)$ and assume that every trajectory $\tilde{\xi}_{x}$ (see (RS.E) of Def. 3) is a Krasowskii Cone Robust solution. Given a cell $P_{1}$ of type I, for every $x \in P_{1}$ we let $\mathcal{K}(x)$ be the Robustness Cone as in Definition 9 associated to $\xi_{x}$ (defined in Def. 3 (RS.B)). Given a cell $P_{2}$ of type II, for every $x \in P_{2}$ and every $P \in \Sigma\left(P_{2}\right)$ we let $\mathcal{K}_{P}(x)$ be the Robustness Cone as in Definition 9 associated to 

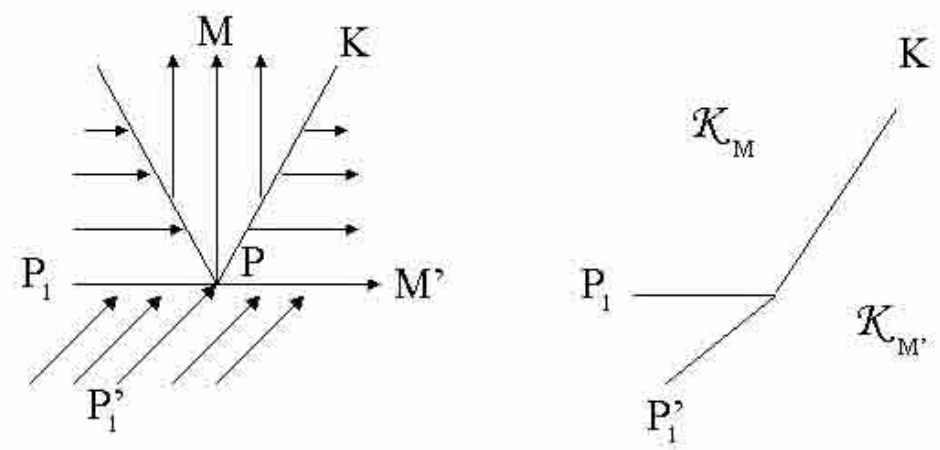

Figure 4. Example of synthesis.

$\xi_{x}^{P}$ (defined in Def. 3 (RS.C)). Moreover, we define $\mathcal{K}_{I I}(x)$ to be the maximal cone for which there exists $a>0$ such that for every $y_{n} \rightarrow x(t), y_{n}=x+\epsilon_{n} w+o\left(\epsilon_{n}\right)$ with $w \in \mathcal{K}_{I I}(x)$ and $\epsilon_{n} \rightarrow 0$, there exist $x_{n}(\cdot)$ Krasowskii solutions with $x_{n}(0)=y_{n}$ and $P \in \Sigma\left(P_{2}\right)$ such that $x_{n}$ converge to $\xi_{x}^{P}$ uniformly on $[0, a]$.

Remark. Roughly speaking, $\mathcal{K}_{I I}(x)$ is the Cone of Weak Robustness for the collection of Stratified solutions $\left\{\xi_{x}^{P}: P \in \Sigma\left(P_{2}\right)\right\}$ with the meaning that $\mathcal{K}_{I I}(x)$ can be taken as Robustness Cone if we replace in Definition 9 the condition that "every sequence of Krasowskii solution $x_{n}$ from $y_{n}$ converge to $x$ " with the condition "there exists a sequence of Krasowskii solution $x_{n}$ from $y_{n}$ converging to $x$ ".

We are now ready to give the following:

Definition 13. The regular synthesis $\Xi=\left(\mathcal{P}, \mathcal{P}_{1}, \mathcal{P}_{2}, \Pi, \Sigma, u\right)$ is Krasowskii admissible if the following holds (with the notation of Def. 12).

H) Each trajectory $\tilde{\xi}_{x}$ (see $(R S . E)$ of Def. 3 ) is a Krasowskii Cone Robust solution.

HI) If $P_{1}$ is a cell of type I then

$\mathbf{H I} \alpha)$ For every $x \in P_{1}$ there exists a cone $\mathcal{K}_{I}(x)$ such that $x+\mathcal{K}_{I}(x) \subset\left\{x: x \in P, P \in \mathcal{P}_{1}\right\}$ and $V_{K}(x) \subset \operatorname{Int}\left(\mathcal{K}(x) \cup \mathcal{K}_{I}(x)\right)$.

HI $\beta$ ) For each cell $M$ of type I such that $\partial M \supset P_{1}$ the vector field $f(x, u(x))$ on $M$ can be prolonged continuously to $P_{1}$ and we call $v_{M}$ the obtained vector field on $P_{1}$. For each $x \in P_{1}$ let $N(x)$ be the space normal to $T_{x} P_{1}$ then there exists $w \in N(x)$ such that for every sequence $\left\{y_{n}\right\}$ in $M$ with $y_{n} \rightarrow x$, if $\omega=\lim _{n} \frac{x-y_{n}}{\left\|x-y_{n}\right\|}$ then $v_{M}(x) \cdot w>0$ and $\omega \cdot w \geq 0$.

HII) If $P_{2}$ is of type II then for every $x \in P_{2}$

$$
V_{K}(x) \subset \operatorname{Int}\left(\bigcup_{P \in \Sigma\left(P_{2}\right)} \mathcal{K}_{P}(x) \cup \mathcal{K}_{I I}(x)\right)
$$

Remark. Condition $\mathrm{HI} \beta$ ) requires that the limiting field $v_{M}$, from cells $M$ of type I, points into the cell $P_{1}$.

In Figure 4 we represent an optimal synthesis to illustrate assumption HII). The cell of type II under consideration is the point $P$. We have $\Sigma(P)=\left\{M, M^{\prime}\right\}$ where $M$ and $M^{\prime}$ are the support of the two trajectories exiting $P$ vertically and horizontally, respectively. Let $P_{1}$ and $P_{1}^{\prime}$ be the type I cells such that $\Pi\left(P_{1}\right)=\Pi\left(P_{1}^{\prime}\right)=P$ as in Figure 4. $K$ is the II cell from which trajectories start parallel to $M$ and $M^{\prime}$. Consider a sequence of points $y_{n}$ that tends to $P$ staying in the open region bounded by $P_{1}, K$ and containing $M$. Then the trajectories starting from $y_{n}$ converge to the stratified trajectory that runs on $P_{1}$ and then on $M$. Hence we get that $\mathcal{K}_{M}$ is the 
open region bounded by $P_{1}, K$ and containing $M$. In a similar manner it is easy to check that $\mathcal{K}_{M^{\prime}}$ is the open region enclosed by $P_{1}^{\prime}$ and $K$, containing $M^{\prime}$. Moreover, if we take a sequence $y_{n}$ tending to $x$ along $K$ and let $x_{n}$ be trajectories from $y_{n}$, then clearly each $x_{n}$ runs either parallel to $M$ or parallel to $M^{\prime}$. Then every converging subsequence of $x_{n}$ converge either to the trajectory running on $M$ or to that running on $M^{\prime}$. So we get $\mathcal{K}_{I I}=K \cup \mathcal{K}_{M} \cup \mathcal{K}_{M^{\prime}}$.

Regarding the robustness of Stratified Solutions we have the following:

Lemma 1. Let $\Xi=\left(\mathcal{P}, \mathcal{P}_{1}, \mathcal{P}_{2}, \Pi, \Sigma, u\right)$ be a regular synthesis such that assumption HI) and HII) of Definition 13 hold. Let $P$ be a cell of type $I, x \in P$ and $\xi_{x}$ as in (RS.B) of Definition 3. Assume that there exists a cell $M$ of type $I, \operatorname{dim}(M)=n$, such that $\partial M \supset P$, the feedback u restricted to $M$ can be prolonged to a smooth feedback $\tilde{u}$ on $\mathbb{R}^{n}$ and $\xi_{x}$ is a trajectory of $f(x, \tilde{u}(x))$. Then $\xi_{x}$ is Krasowskii Cone Robust at time 0.

Proof. We can apply Theorem 1 with $f(\cdot, \tilde{u}(\cdot))$ playing the role of $v$. The cone $\mathcal{K}$ is here any cone with non empty interior such that $x+\mathcal{K} \subset M$.

Remark. The same conclusion of the Lemma holds also if trajectories from $M$ enters $P$. Thus, in many cases, condition H) of Definition 13, for cells of type I, is indeed a consequence of conditions HI). Therefore we have to ensure condition $\mathrm{H}$ ) only for cells of type II.

\subsection{Main result}

Theorem 3. Let $\Xi=\left(\mathcal{P}, \mathcal{P}_{1}, \mathcal{P}_{2}, \Pi, \Sigma, u\right)$ be a Krasowskii admissible regular synthesis then Krasowskii Cone Robust solutions (not passing through the origin) coincide with Stratified solutions.

Proof. We first prove by induction on codimension that on cells of type I Krasowskii Cone Robust solutions coincide with Stratified ones. Assume that $P_{1}$ is a cell of type I of maximal dimension then $V_{K}=f(\cdot, u(\cdot))$ on $P_{1}$ and the conclusion is trivial.

We now prove the induction step. Let $P_{1}$ be a cell of type I of codimension $r$ and assume that the conclusion holds up to codimension $r-1$.

Consider a point $x \in P_{1}$. From condition (RS.1) of Definition 3, there exists a neighborhood $\mathcal{O}$ of $x$ such that a finite number of cells intersect $\mathcal{O}$ and the closure of each of them intersect $P_{1}$. Then, from the first condition of Definition 2, all of them, except $P_{1}$, have dimension strictly greater than the dimension of $P_{1}$.

Assume, by contradiction, that there exists a Krasowskii Cone Robust solution $\eta:[0, \epsilon] \rightarrow \mathbb{R}^{n}$ with $\eta(0)=x$ such that for every $\epsilon^{\prime}>0$ there exists $\left.\left.t \in\right] 0, \epsilon^{\prime}\right]$ for which $\eta(t) \neq \xi_{x}(t)$. From assumption HI $\alpha$ ) and the semicontinuity of $V_{K}$, we have that either there exists a strictly decreasing sequence $t_{n} \rightarrow 0$ such that $\eta\left(t_{n}\right) \in$ $x+\mathcal{K}(x)$, or $\eta\left(\left[0, \epsilon^{\prime}\right]\right) \subset \bigcup\left\{y: y \in P, P \in \mathcal{P}_{1}\right\}$ for some $0<\epsilon^{\prime}<\epsilon$.

If the former case happens, define $\hat{\eta}_{n}$ to be the restriction of $\eta$ to the interval $\left[t_{n}, t_{n}+\epsilon\right]$. Passing to a subsequence we can assume that there exists $w \in \mathcal{K}(x)$ such that $\hat{\eta}_{n}\left(t_{n}\right)=x+\epsilon w+o\left(\epsilon_{n}\right)$ with $\epsilon_{n} \rightarrow 0$. Then by assumption $\mathrm{H})$ and the definition of $\mathcal{K}(x)$, there exists $a>0$ such that $\hat{\eta}_{n}$ restricted to $\left[t_{n}, t_{n}+a\right]$ converge uniformly to $\xi_{x}$ obtaining a contradiction. Hence, from now on we assume to be in the latter case.

Assume that $\eta\left(\left[0, \epsilon^{\prime \prime}\right]\right) \subset P_{1}$ for some $\epsilon^{\prime \prime}>0$. Since $\eta$ is Cone Robust there exists a sequence of Krasowskii solutions $\eta_{n}$ converging to $\eta$ on $\left[0, a^{\prime}\right]$ for some $0<a^{\prime}<\epsilon^{\prime \prime}$. Now, from condition HI $\alpha$ ) and the upper semicontinuity of $V_{K}$, we have that for $n$ sufficiently big $\eta_{n}$ eiher enters $x+\mathcal{K}(x)$ or the set $\left\{y: y \in P, P \in \mathcal{P}_{1}\right\}$. In the former case we would have that $\eta_{n}$ converge to $\xi_{x}$ obtaining a contradiction.

Assume the latter case and take $t \in] 0, a^{\prime}[$ such that $\dot{\eta}(t) \neq f(\eta(t), u(\eta(t)))$. There exists a cell $M$ of type I such that $\eta_{n}(t) \in M$ for infinitely many $n$.

If $M=P_{1}$ we let $\tilde{\eta}_{n}$ be the concatenation of $\eta_{n}$ restricted to $[0, t]$ with the trajectory $\xi_{\eta_{n}(t)}$ restricted to $\left[t, a^{\prime}\right]$. Then $\tilde{\eta}_{n}$ can not converge to $\eta$. Indeed the right derivatives in $t$ of $\tilde{\eta}_{n}$ converge to $f(\eta(t), u(\eta(t)))$ obtaining a contradiction.

If otherwise $M \neq P_{1}$, let $\tau_{n}=\sup \left\{s \in\left[t, a^{\prime}\right]: \eta_{n}(\sigma) \in M\right.$ for all $\left.\sigma \in[t, s]\right\}$. Since $M$ is of type I, by induction, we have $\tau_{n}>t$. If $\tau_{n}-t>\delta>0$ for infinitely many $n$ then we contradict assumption $\left.\mathrm{HI} \beta\right)$. 
Otherwise, there exists $M^{\prime}$ of type I such that $\eta_{n}\left(\tau_{n}\right) \in M^{\prime}$ for infinitely many $n$. Since $\operatorname{cl}(M) \cap M^{\prime} \neq \emptyset$, from (RS.1) of Definition 3, we get $\operatorname{dim}\left(M^{\prime}\right)<\operatorname{dim}(M)$. Now we can reason for $M^{\prime}$ as for $M$. After a finite number of steps we obtain that $\eta_{n}$ enters $P_{1}$ again, reaching a contradiction, as in the previous case.

Assume now that for every $\delta>0, \eta([0, \delta])$ is not entirely contained in $P_{1}$. If $\eta(] 0, \delta[) \subset M$ for some $\delta>0$ and $M \neq P$, then we contradict assumption $\mathrm{HI} \beta)$. Indeed, using the notation of $\mathrm{HI} \beta$ ) of Definition 13 , we define $\varphi(t) \stackrel{\text { def }}{=} v \cdot(\eta(t)-x)$. Then $\varphi$ is absolutely continuous, $\varphi(0)=0$ and for $t$ sufficiently small $\dot{\varphi}(t)>0$ and $\varphi(t)<0$. Therefore we obtain a contradiction.

Otherwise, there exists a cell $M$ and a strictly decreasing sequence $t_{n}$ converging to zero such that $\eta\left(t_{n}\right) \in M$ and for every $n$ there exists $s \in] t_{n+1}, t_{n}$ [ such that $\eta(s) \notin M$. Taking $n$ sufficiently big we can assume that $\eta\left(\left[0, t_{n}\right]\right) \subset \mathcal{O}$ and we define:

$$
\tau=\inf \left\{t \in\left[0, t_{n}\right]: \eta(s) \in M \forall s \in\left[t, t_{n}\right]\right\}
$$

We have that $\tau>t_{n+1}>0$. Moreover $M$ is of type I, hence, by induction, the only Krasowskii solution from $\eta\left(t_{n}\right)$ stays in $M$ in a neighborhood of $t_{n}$ so $\tau<t_{n}$.

If $\eta(\tau) \in M^{\prime}$ with $M^{\prime} \neq M$ then $M^{\prime} \cap \operatorname{cl}(M) \neq \emptyset$ hence $\operatorname{dim}\left(M^{\prime}\right)<\operatorname{dim}(M)$. Now if $M^{\prime}=P_{1}$ then we contradict $\mathrm{HI} \beta$ ), otherwise we contradict the induction hypothesis.

If $\eta(\tau) \in M$ then there exists a strictly increasing sequence $\sigma_{n}, \sigma_{n} \rightarrow \tau, \eta\left(\sigma_{n}\right) \in M^{\prime}$ with $M^{\prime}$ of type I, hence $\operatorname{cl}\left(M^{\prime}\right) \cap M \neq \emptyset$ so $\operatorname{dim}\left(M^{\prime}\right)>\operatorname{dim}(M)$. If this is the case, we can reason for $M^{\prime}$ as for $M$. But this can happen only a finite number of times. This concludes the inductive step.

Let now $P_{2}$ be a cell of type II and $x \in P_{2}$. From condition HII) and the upper semicontinuity of $V_{K}$, there exists $\epsilon>0$ such that for every $y$ with $|y-x|<\epsilon$

$$
V_{K}(y) \subset \mathcal{K}^{\prime} \stackrel{\text { def }}{=} \operatorname{Int}\left(\bigcup_{P \in \Sigma\left(P_{2}\right)} \mathcal{K}_{P}(x) \cup \mathcal{K}_{I I}(x)\right) .
$$

Let $\eta$ be a Krasowskii Cone Robust Solution starting at $x$ and assume that there exists no $P \in \Sigma\left(P_{2}\right)$ such that $\eta=\xi_{x}^{P}$ on some interval $\left[0, \epsilon^{\prime}\right], \epsilon^{\prime}>0$. From the robustness of $\eta$ there exist $y_{n} \rightarrow x$ and Krasowskii Solutions $\eta_{n}$, with $\eta_{n}(0)=y_{n}$, converging uniformly to $\eta$ on some interval $[0, a], a>0$. By equation $(7)$, since $y_{n} \rightarrow x$, for $n$ sufficiently big there exists $\epsilon_{n}$ such that $\epsilon_{n} \rightarrow 0$ and $\eta_{n}\left(\epsilon_{n}\right) \in x+\mathcal{K}^{\prime}$.

If there exists $P \in \Sigma\left(P_{2}\right)$ such that, for infinitely many $n, \eta_{n}\left(\epsilon_{n}\right) \in x+\mathcal{K}_{P}(x)$ then $\eta_{n}$ converges to $\xi_{x}^{P}$ obtaining a contradiction.

If otherwise, for infinitely many $n, \eta_{n}\left(\epsilon_{n}\right) \in x+\mathcal{K}_{I I}(x)$ then there exist $P \in \Sigma\left(P_{2}\right)$ and Krasowskii Solutions $\hat{\eta}_{n}$, with $\hat{\eta}_{n}(0)=\eta_{n}\left(\epsilon_{n}\right)$, such that $\hat{\eta}_{n}$ converges to $\xi_{x}^{P}$. Define $\tilde{\eta}_{n}$ to be the concatenation of $\hat{\eta}_{n}$ (time shifted) with $\eta_{n}$ restricted to $\left[0, \epsilon_{n}\right]$. Then $\tilde{\eta}_{n}$ converge to $\xi_{x}^{P}$ and contradict the robustness of $\eta$.

This concludes the proof of the theorem.

Remark. For generic planar systems the set of Caratheodory solutions coincides with the set of Stratified solutions as seen in the previous section. Moreover the proof of Theorem 3 for cells of type I can be applied to Caratheodory solutions. Indeed Caratheodory solutions are Krasowskii solutions and the proof applies to Krasowskii solutions except for the case in which the considered trajectory $\eta$ lies entirely on the cell $P_{1}$, when the Cone Robustness of $\eta$ is used. However, it is clear that there is only one Caratheodory solution lying on $P_{1}$, namely the Stratified solution.

In general we do not expect Caratheodory solutions to coincide with Krasowskii Cone Robust and Stratified ones as shown in next example.

Example. Consider the synthesis depicted in Figure 5. The set of Stratified Solutions through the cell of type II, $P$, is given by two trajectories: one running on $M_{1}$ and then on $M_{2}$, the other running on $M_{1}$ and then on $M_{3}$. It is easy to check that the set of Krasowskii Cone Robust Solutions coincide with the set of Stratified 


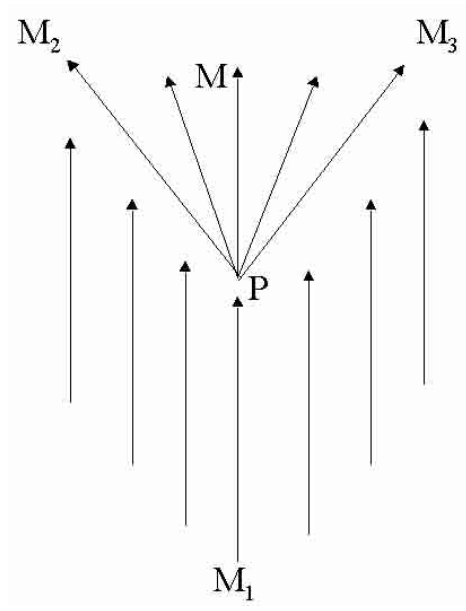

FiguRE 5. Example of synthesis.

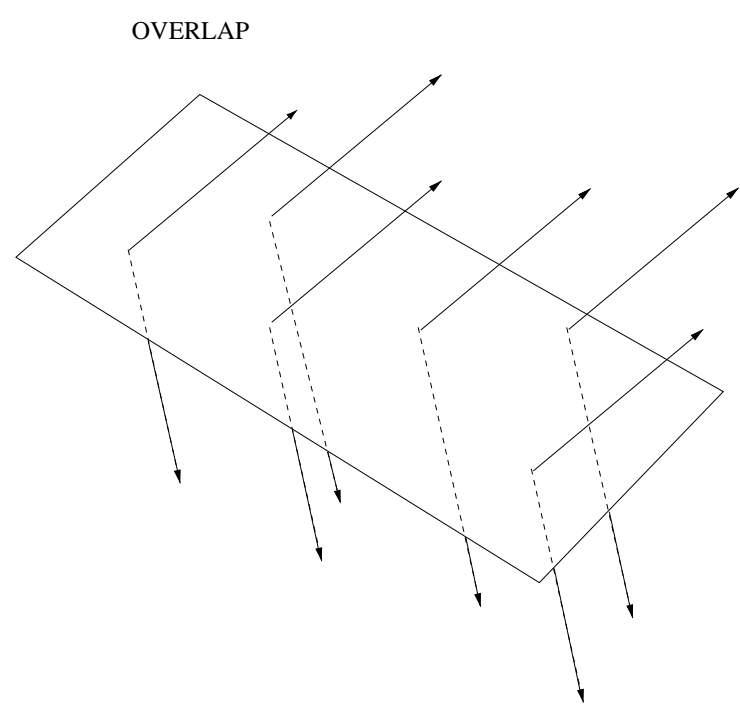

FiguRE 6. Overlap hypersurface.

ones. The set of Caratheodory Solutions through $P$ contains also the trajectories running on $M_{1}$ and then entering the two dimensional cell $M$.

\subsection{Overlaps}

In Figure 6 we represent an overlap hypersurface that typically occurs in optimal syntheses, see [11,16]. From each point of this hypersurface we have two Stratified trajectories starting towards opposite sides of the hypersurface. Notice that both $V_{K}$ and $V_{F}$ on the hypersurface contain a direction tangent to it. Thus Krasowskii and Filippov solutions strictly contain the stratified ones. However a Krasowskii (Filippov) solution $\xi$ running on this hypersurface can not be Cone Robust. Indeed for every cone $\mathcal{K}$ with nonempty interior, there exists a sequence $\left\{x_{n}\right\}$ of points tending to the initial point of $\xi$ that are outside the hypersurface. Thus 

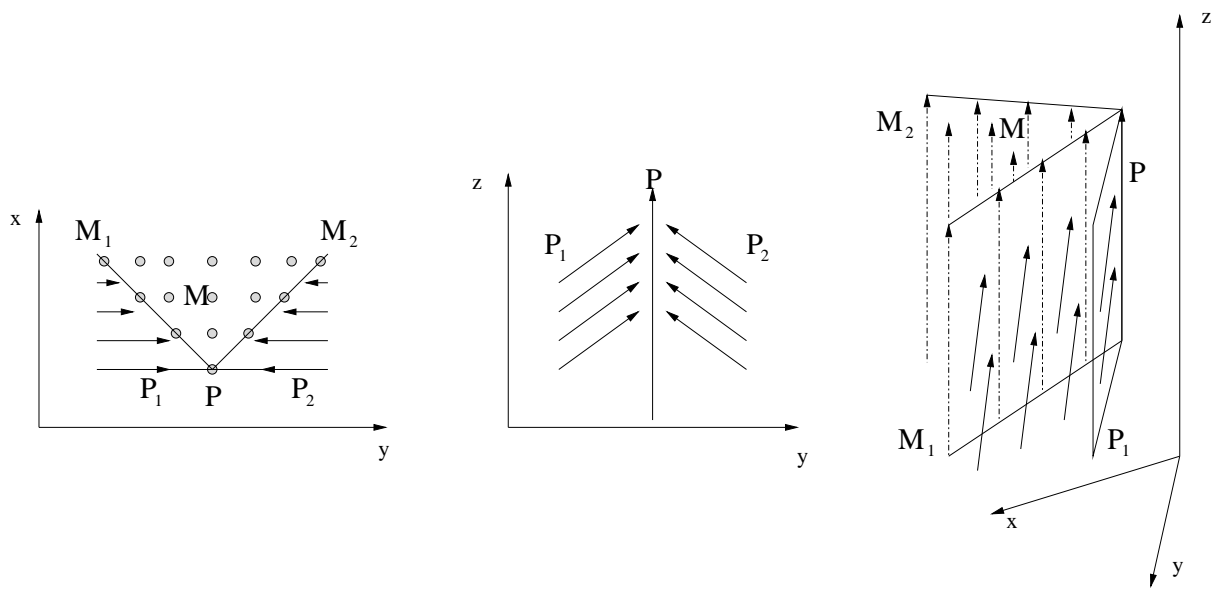

Figure 7. Example of synthesis.

every Krasowskii solution $\xi_{n}$ starting from $x_{n}$ is completely outside the hypersurface. It easily follows that the sequence $\xi_{n}$ can not converge to $\xi$.

\subsection{Stratified solutions}

We want to discuss the assumptions of Theorem 3.

The assumption H) is obviously necessary to guarantee the validity of the theorem. It is not clear if this can hold for general systems, however, as we saw in Section 4, it is true for minimum time on the plane (for single input systems). Moreover, we observed that on cells of type I, under suitable assumptions, H) follows from conditions $\mathrm{HI}$ ).

Notice that to violate $\mathrm{H}$ ) on a cell $P$ of type I, we should have a Krasowskii Cone Robust solution that is not a Stratified one, starting from a point $x$ of $P$. We give below further conditions under which there is no trajectory running on $P$ but the Stratified one.

Proposition 2. Let $\Xi=\left(\mathcal{P}, \mathcal{P}_{1}, \mathcal{P}_{2}, \Pi, \Sigma, u\right)$ be a Krasowskii admissible regular synthesis. Consider a cell $P$ of type $I$ and assume that all neighboring cells are of type $I$. Then for every $x \in P, V_{K}(x) \cap T_{x} P=f(x, u(x))$.

Proof. Assume by contradiction that there exists a control $\omega$ such that $f(x, \omega) \in V_{K}(x) \cap T_{x} P$ and $f(x, \omega) \neq$ $f(x, u(x))$. Since $f(x, \omega)$ is obtained as convex combination of limits of $f(x, u(x))$ along I cells, we can extend $\omega$ to a feedback defined on a neighborhood of $x$ in $P$ in a smooth way. By assumption $\mathrm{H}$ ), for every sequence $y_{n} \in \mathcal{K}(x), y_{n} \rightarrow x$ and $x_{n}$ Krasowskii solutions starting at point $y_{n}, x_{n}$ converge uniformly to $\xi_{x}$. There exists a I cell $M$ such that $y_{n} \in M$ for infinitely many $n$. Using $\mathrm{HI} \beta$ ) and reasoning as in the proof of Theorem 3 we get that $x_{n}$ enter $P$. Then $x_{n}$ could use the control $\omega$ contradicting $\left.\mathrm{H}\right)$.

Observe that, Proposition 2 is based on condition $\mathrm{HI} \beta$ ). If we relax this condition then singularities on the synthesis, as in Figure 7, may arise. In this case the conclusion of Proposition 2 is still guaranteed if the following condition is required:

$\mathrm{HI} \tilde{\beta})$ The assumption $\mathrm{HI} \beta$ ) holds for every cell $P$ of type I with the condition $v_{M}(x) \cdot w>0$ replaced by $v_{M}(x) \cdot w \geq 0$. Let $P$ be a cell of type I of codimension $r$ then, for every $x \in P, \mathcal{K}(x)$ contains a subspace of dimension $r$.

Proposition 3. Let $\Xi=\left(\mathcal{P}, \mathcal{P}_{1}, \mathcal{P}_{2}, \Pi, \Sigma, u\right)$ be a Krasowskii admissible regular synthesis with condition HI $\left.\beta\right)$ replaced by HI $\tilde{\beta})$. Consider a cell $P$ of type I of codimension $r$ and assume that all neighboring cells are of type I. Then for every $x \in P, V_{K}(x) \cap T_{x} P=f(x, u(x))$. 
Proof. Assume by contradiction that there exists a control $\omega$ such that $f(x, \omega) \in V_{K}(x) \cap T_{x} P$ and $f(x, \omega) \neq$ $f(x, u(x))$. Since $f(x, \omega)$ is obtained as convex combination of limits of $f(x, u(x))$ along I cells, there exist two cells $P^{\prime}$ and $P^{\prime \prime}$ of type I such that the convex hull of limits of $f(x, u(x))$ along these cells contains $f(x, \omega)$ and the following holds. Define the limiting tangent spaces $\Pi^{\prime}$ and $\Pi^{\prime \prime}$ by $\Pi^{\prime}=\lim _{y \rightarrow x, y \in P^{\prime}} T_{y} P^{\prime}$ and $\Pi^{\prime \prime}=\lim _{y \rightarrow x, y \in P^{\prime \prime}} T_{y} P^{\prime \prime}$. Then, by condition (RS.1) of Definition $3, \Pi^{\prime} \cap \Pi^{\prime \prime}$ strictly contains $T_{x} P$ hence $\operatorname{dim}\left(\Pi^{\prime} \cap \Pi^{\prime \prime}\right)>n-r$. Therefore $(x+\mathcal{K}(x)) \cap\left(P^{\prime} \cup P^{\prime \prime}\right) \neq \emptyset$. If $y_{n} \in(x+\mathcal{K}(x)) \cap\left(P^{\prime} \cup P^{\prime \prime}\right)$ and $x_{n}$ are Krasowskii solutions starting at $y_{n}$ then $x_{n}$ enter $P$. Hence $x_{n}$ could use the control $\omega$ contradicting $\left.\mathrm{H}\right)$.

\subsection{Extremal solutions}

In this section we want to discuss the role of Krasowskii Extremal solutions in cases where the conclusion of Proposition 2 does not hold.

Assume that $P$ is a cell of type I and $V_{K}(x) \cap T_{x} P \supset f(x, u(x))$ where the inclusion is strict. We can try to isolate Stratified Solutions looking for Extremal Krasowskii Solutions.

Definition 14. Let $\Xi=\left(\mathcal{P}, \mathcal{P}_{1}, \mathcal{P}_{2}, \Pi, \Sigma, u\right)$ be a regular synthesis on $\Omega$. Given a point $x \in \Omega$ let $K r C R(x)$ be the set of Krasowskii Cone Robust Solutions through $x$. Assume that each $\xi \in \operatorname{KrCR}(x)$ is differentiable from the right at time $t$ with $\xi(t)=x$ and define

$$
R_{K}(x) \stackrel{\text { def }}{=}\left\{D^{+} \xi(t): \xi \in K r C R(x), \xi(t)=x\right\} .
$$

Proposition 4. Let $\Xi=\left(\mathcal{P}, \mathcal{P}_{1}, \mathcal{P}_{2}, \Pi, \Sigma, u\right)$ be a regular synthesis on $\Omega, P$ a cell of type $I$ and $x \in P$ then:

a. if $w \in V_{K}(x) \cap T_{x} P, w \neq f(x, u(x))$ then $w \notin \operatorname{ext} V_{K}(x)$;

b. $\operatorname{ext} R_{K}(x) \supset \operatorname{ext} V_{K}(x) \cap R_{K}(x)$.

Proof. Assume that $w \in V_{K}(x) \cap T_{x} P$ and $w \neq f(x, u(x))$ then $w$ is obtained as convex combination of limits of $f(\cdot, u(\cdot))$ restricted to neighboring cells of type I. Hence $w$ can not be an extremal point of $V_{K}(x)$. Thus a. is proved.

The conclusion b. follows from the fact that if $w$ is an extremal point of $V_{K}(x)$ then it is also an extremal point of the smaller set $R_{K}(x)$.

We can give suitable conditions in such a way that Extremal Solutions help in selecting Stratified solutions.

Proposition 5. Let $\Xi=\left(\mathcal{P}, \mathcal{P}_{1}, \mathcal{P}_{2}, \Pi, \Sigma, u\right)$ be a regular synthesis on $\Omega, P$ a cell of type I. Assume that, for every $x \in P, f(x, u(x)) \in R_{K}(x)$. Moreover, either

i. $R_{K}(x) \cap T_{x} P=f(x, u(x))$ for every $x \in P$, or

ii. $f(x, u(x)) \in \operatorname{ext} V_{K}(x)$ for every $x \in P$.

Then Stratified Solutions are either the only Krasowskii Cone Robust Solutions contained in $P$ or the only Krasowskii Cone Robust Solutions that are extremal and contained in P.

Proof. We have that Stratified Solutions are Krasowskii Cone Robust. If i. holds then Stratified Solutions are clearly the only Krasowskii Cone Robust Solutions contained in $P$. If otherwise ii. holds then Stratified Solutions are extremal and we conclude by a. of Proposition 4.

\section{REFERENCES}

[1] J.P. Aubin and A. Cellina, Differential Inclusions. Springer-Verlag (1984).

[2] A. Bicchi, A. Ballucchi, B. Piccoli and P. Soueres, Stability and robustness of optimal synthesis for route tracking by dubins' vehicles, in Proc. IEEE Int. Conf. on Decision and Control (2000).

[3] V.G. Boltyanskii, Sufficient conditions for optimality and the justification of the dynamic programming principle. SIAM J. Control Optim. 4 (1966) 326-361.

[4] U. Boscain and B. Piccoli, Extremal synthesis for generic planar systems. J. Dynam. Control Systems 7 (2001) $209-258$. 
[5] A. Bressan and B. Piccoli, A generic classification of time-optimal planar stabilizing feedbacks. SIAM J. Control Optim. 36 (1998) 12-32.

[6] P. Brunovsky, Existence of regular syntheses for general problems. J. Differential Equations 38 (1980) $317-343$.

[7] F.H. Clarke, Y.S. Ledyaev, E.D. Sontag and A.I. Subbotin, Asymptotic controllability implies feedback stabilization. IEEE Trans. Automat. Control 42 (1997) 1394-1407.

[8] A.F. Filippov, Differential Equations with Discontinuous Righthand Sides, Mathematics and Its Applications (Soviet Series). Kluwer Academic Publishers Group (1988). Translated from the Russian.

[9] C.G. Gibson, Construction of canonical stratifications, in Topological Stability of Smooth Mappings. Springer Verlag, Berlin, Lecture Notes in Math. 552 (1976) 9-34

[10] M. Goresky and R. MacPherson, Stratified Morse Theory. Springer Verlag, Berlin (1988).

[11] A.J. Krener and H. Schättler, The structure of small time reachable sets in low dimensions. SIAM J. Control Optim. 27 (1989) $120-147$.

[12] J.P. Laumond and P. Souères, Shortest paths synthesis for a car-like robot. IEEE Trans. Automat. Control 41 (1996) $672-688$.

[13] A. Marigo and B. Piccoli, Safety controls and applications to the dubins' car. Nonlinear Differential Equations and Applications (in print).

[14] A. Marigo and B. Piccoli, Safety driving for the dubins' car, in XV World Congress on Automatic Control b'02 (in print).

[15] R. Murray, Nonlinear control of mechanical systems: A Lagrangian perspective, in IFAC Symposium on Nonlinear Control Systems Design (NOLCOS) (1995) 378-389.

[16] B. Piccoli, Classification of generic singularities for the planar time optimal syntheses. SIAM J. Control Optim. 34 (1996) 914-1946.

[17] B. Piccoli and H.J. Sussmann, Regular synthesis and sufficiency conditions for optimality. SIAM J. Control Optim. 39 (2000) 359-410.

[18] H.J. Sussmann, Regular synthesis for time-optimal control of single-input real analytic systems in the plane. SIAM J. Control Optim. 25 (1987) 1145-1162. 